\title{
Studies on the Generative Nuclear Division II. Behavior of the chromosomes during the generative nuclear division in Allium fistulosum
}

\author{
Akio Kamizyô and Nobunori Tanaka \\ Botanical Laboratories, School of Medicine, Teikyo University, \\ 359 Ôtsuka, Hachiôji City, Tokyo, 192-03 Japan
}

Received February 15, 1980

In most angiosperms with binucleated pollen grains, the division of the generative nucleus is carried out in the developing pollen tube. Behavior of metaphasic chromosomes of this division has been studied by many investigators (Maheshwari 1949, review, Ôta 1957). In most plants, the kinetochores of metaphasic chromosomes arrange in the equatorial plate in somatic mitosis, including pollen tube mitosis as reported by Cooper (1936). However, when the space for mitosis is limited or too small to allow free behavior of chromosomes, especially of large chromosomes, some disturbance may take place in the course of mitosis. In Allium odorum, chromosomes are seen much crowded in a pollen tube nuclear division, and it has been explained as large chromosomes were forced to arrange in the narrow metaphase plate, so they are inevitably jammed all together (Ôta 1957). But, no further detailed observation concerned to the process of chromosomal transformation and transition through prophase to metaphase in the pollen tube nuclear division has been described by the author.

In somatic mitosis, on the other hand, it has been said for long time, by many investigators, that a topographical chromosomal configuration in telophase persists through interphase to prophase of the next mitosis without any definite nor comprehensive tracing experiments. In recent years, however, this situation has been much improved by the use of Giemsa $\mathrm{C}$-banding technique, and in some cases, this phenomenon has been recognized to be true to a certain extent in Allium cepa (Stack and Clark 1974, Roy and Ghosh 1977, Ghosh and Roy 1977), and in some Polygonatum species (Tanaka and Tanaka 1977).

In our previous paper, in the root-tip cells of Allium fistulosum, the presence of clear C- and Q-bands has been found in the satellite regions of SAT chromosomes as well as in all distal ends of almost all chromosomes. The same situation has also been observed in the pollen tube nuclear division (Kamizyô and Tanaka 1978).

Further study on the behavior of chromosomes in the generative nuclear division in Allium fistulosum, which has been carried out by means of C-banding technique and of aceto-orcein staining, will be described here. 


\section{Materials and methods}

The materials used in this study were taken from the same source as used in our previous study, but the methods applied were slightly modified as follows. No colchicine was added to the culture medium except as otherwise stated later in "results". Cultured pollen tubes were fixed after proper time lapses by pouring the fixing agent into the culture vial. Thus prepared mixed solution contained a number of pollen grains at various developing stages, according to the time how long the pollen grains were cultured. One drop of the fixed pollen grain suspension was placed on a slide glass and air dried completely enough to prevent the materials drop off from the preparation. Thus made preparations were then hydrolysed, washed with distilled water, and air dried again. These hydrolysed materials were processed for C-banding technique.

When to make aceto-orcein preparations, dried preparations were stained directly with aceto-orcein without hydrolysis. After microscopical observation, a part of the aceto-orcein stained preparations were processed to C-banding technique; namely, the same cells as photographed were used for C-banding analysis. After the coverslip was separated from the slide glass by dry ice method, the materials were destained with acetic alcohol, hydrolysed, and finally restained by C-banding technique. For comparative observation of somatic chromosomes, root-tip cells were processed to C-banding technique in parallel runs.

Results

\section{1) Somatic chromosomes}

When the chromosomes of root-tip cells of Allium fistulosum were stained by $\mathrm{C}$-banding technique, the deeply stained $\mathrm{C}$-bands have been identified in nearly all telomeres and satellites (Fig. 1). The aspects of the topographical distribution of C-bands in the nuclei at the stages of telophase, interphase and prophase are shown in Figs. 2, 3 and 4, respectively. These figures suggest that, as it has been already reported by many investigators in many plants species, in the root-tip cells of Allium fistulosum, the kinetochores migrate into the equatorial region at metaphase, subsequently the daughter chromosomes separate toward the opposite poles, and thereafter thus formed telophasic configuration is persisted throughout the successive stages of the cell cycle, from interphase to prophase.

\section{2) Generative chromosomes}

The germination of pollen tubes took place usually in $15 \mathrm{~min}$ cultivation. When pollen grains were cultured in the medium containing no colchicine, generative mitotic phases of metaphase through telophase were observed abundantly in the $4 \mathrm{hr}$ culture. As stated in our previous paper, owing to certain difficulties met with in $\mathrm{C}$-banding technique, especially when applied to pollen tube generative nuclei, it does not always give a good contrast figure. A majority of preparations were stained uniformly equally with meagre contrast. Forthermore, since the extine of pollen grains was stained deeply by this technique, it was very difficult to ana- 
lyse nuclear staining reaction of nuclei still nest in the pollen grains.

Before germination of the pollen tube, the generative nucleus has a crescentor spindle-shaped form, and stands at mitotic prophase (Fig. 5). Fig. 6 shows a naked generative nucleus, which happened get free out of the pollen tube in a $30 \mathrm{~min}$ cultured preparation. This naked nucleus shows a typical figure of midprophase in which chromosomal spiremes are seen packed altogether in lengthwise direction as simulated as just packed intact in the real pollen tube. The vegetative

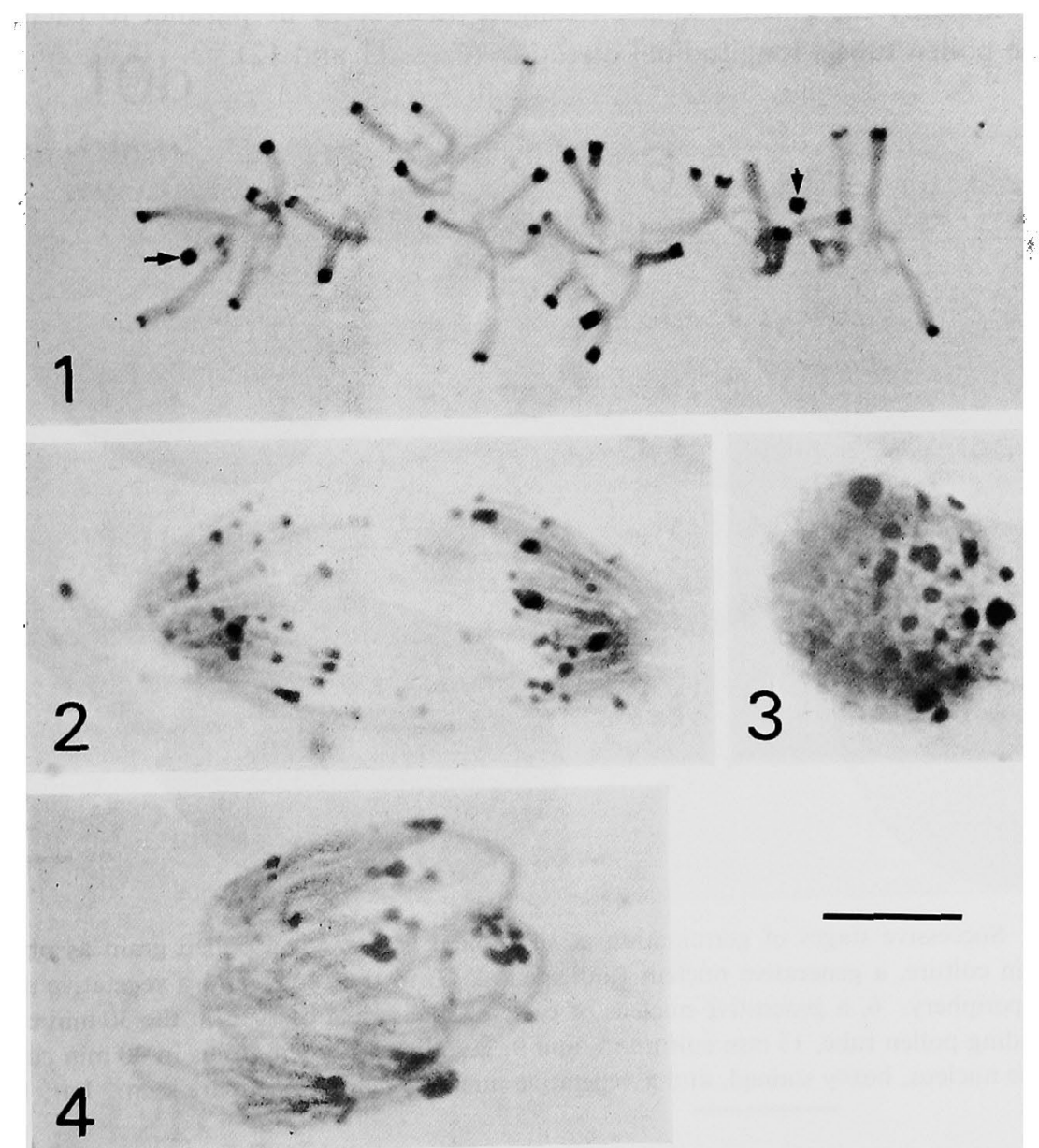

Figs. 1-4. Root-tip nuclei stained with C-banding technique. 1, early anaphase. 2, telophase. 3, interphase. 4, prophase. Arrows indicate satellites. Scale, $10 \mu \mathrm{m}$.

nucleus was always stained weakly with aceto-orcein. And its form varied in various ways; an example of an extremely extended vegetative nucleus was shown in Fig. 19. The generative and the vegetative nuclei proceded into the pollen tube seemingly at random but sometimes associated together. Figs. 7 and 8 show an independent entrance of the two nuclei into the pollen tube. Fig. 9 shows a simultaneous rush of the two nuclei into the pollen tube.

In the $2 \mathrm{hr}$ culture the generative nucleus developed into the mitotic stage of mid-prophase (Fig. 10) or late prophase (Figs. 11 and 12). They are stained with 
aceto-orcein in a-series microphots (Figs. 10a, 11a and 12a) and by C-banding techniques in b-series microphots (Figs. 10b, 11b and 12b). Those figures in the $b$-series microphots are taken from the same nuclei stained in the first by acetoorcein. They clearly demonstrate that, in mid-prophase, the chromosomes are holding a telophasic configuration of the previous mitosis, which is the primary microspore division carried out in the pollen grain (Fig. 10). And that, in late prophase, considerable revolution of a part of chromosomal arms has occurred, and consequently the chromosomes arranged themselves in parallel to each other along the pollen tube's longitudinal direction (Figs. 11 and 12).

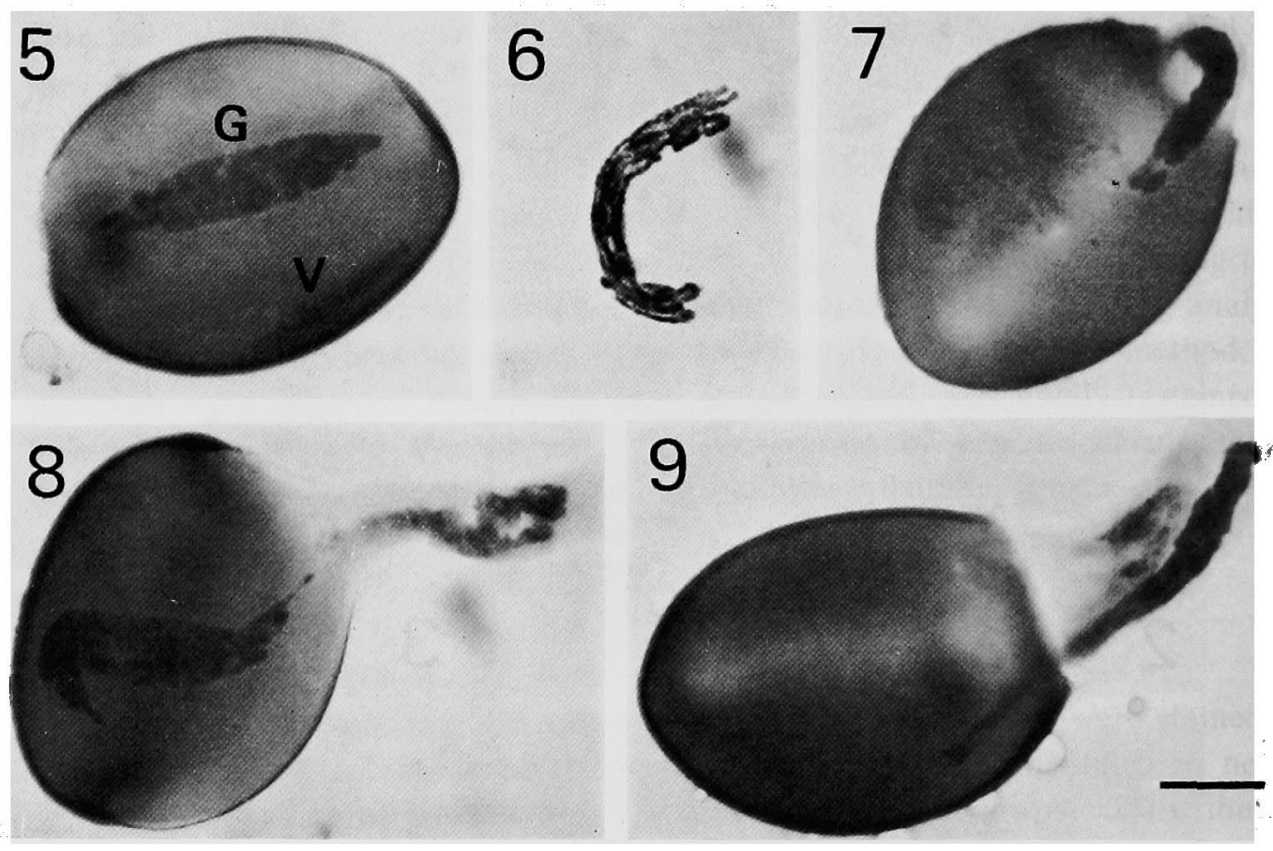

Figs. 5-9. Successive stages of germination of the pollen grains. 5, a pollen grain as observed in the 5 min culture, a generative nucleus (indicated as $G$ ) in the center and a vegetative nucleus (V) in the periphery. 6, a generative nucleus of early prophase as observed in the 30 min culture. 7, a protruding pollen tube, 15 min culture. 8 and 9, germinated pollen grains in 30 min cultures; a generative nucleus, heavy stained, and a vegetative nucleus, light stained, are seen. Bar, $10 \mu \mathrm{m}$.

Fig. 13 shows a prometaphasic nucleus observed in the $4 \mathrm{hr}$ culture. In this nucleus, the chromosomes are just about to arrange themselves in a nuclear plate formed coincidentlly with the pollen tube's longitudinal axis. Thus formed nuclear plate was observed frequently in prometaphasic nuclei as well as in metaphasic ones (Figs. 14 and 15). In these nuclei, the direction of chromosomal arms is not necessarily parallel to the longitudinal axis of the pollen tube. On the other hand, in the nucleus shown in Fig. 16, the chromosomes arrange themselves tightly side-by-side forming a perpendicular nuclear plate against the pollen tube's longitudinal axis. However, owing to the crowdedness of chromosomes, identification of each individual chromosomes is quite impossible. Such nuclear plate formation as shown in Fig. 16, that is a quite normal feature in pollen tube mitotic 


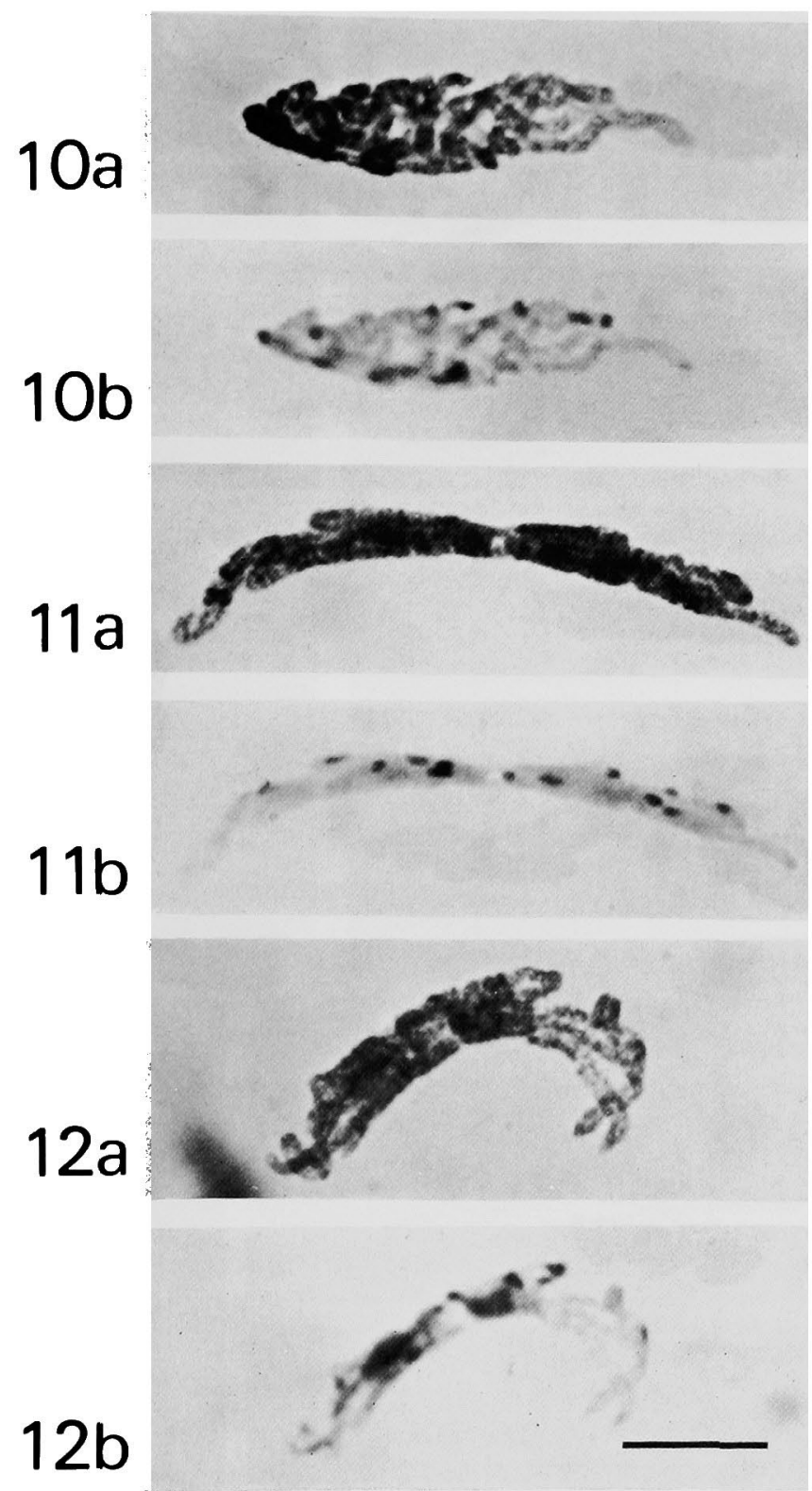

Figs. 10-12. Generative nuclei stained sequentially in one and the same cell. a, aceto-orcein, and $\mathrm{b}, \mathrm{C}$-banding preparation, prepared from the $2 \mathrm{hr}$ culture of the pollen tubes. 10a and 10b, midprophase. $11 \mathrm{a}, 11 \mathrm{~b}, 12 \mathrm{a}$ and $12 \mathrm{~b}$, late prophase. Bar, $10 \mu \mathrm{m}$.

metaphase in other species of plants, was observed not frequently in this material. When pollen grains were cultured in the medium containing $0.02 \%$ colchicine for $6 \mathrm{hr}$, the primary constriction of eight chromosomes and the secondary constriction of a SAT chromosome (Fig. 17) appeared clearly. It is noteworthy to comment here that in these colchicine treated nuclei, the degree of chromosomal condensation and the dispersion of individual chromosomes along the longitudinal 


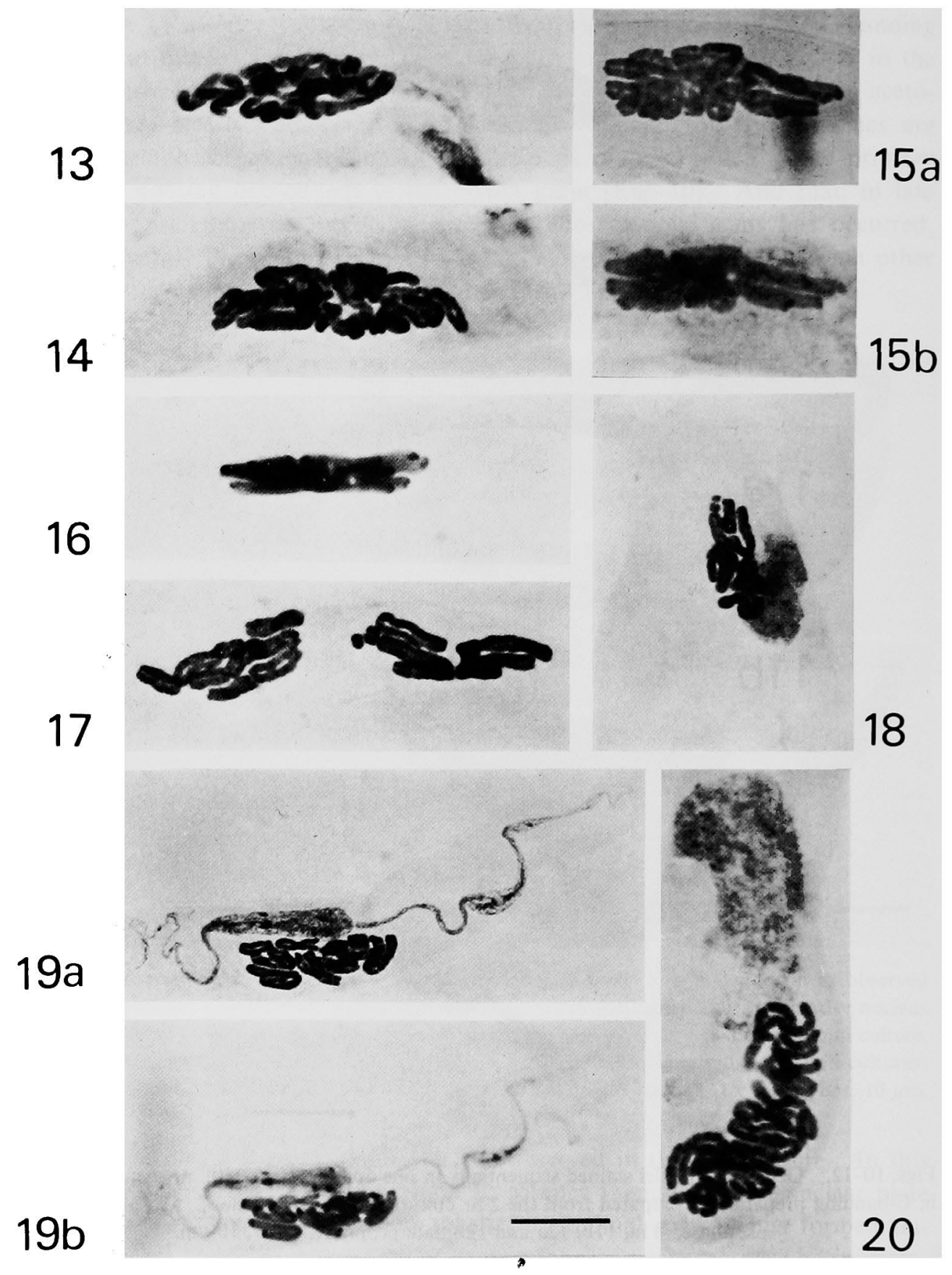

Figs. 13-20. 13, a prometaphasic nucleus as observed in the $4 \mathrm{hr}$ culture and stained with acetoorcein. 14-20: Metaphasic nuclei in various configurations. 14-16, not treated with colchicine. $17-20$, treated with $0.02 \%$ colchicine. 16 , as observed in the $4 \mathrm{hr}$ culture and others, $6 \mathrm{hr}$ culture. 14,15 and 19 are metaphases in polar view and others are that in side view. $15 \mathrm{~b}, 16$ and $19 \mathrm{~b}$ are stained by C-banding technique and others, stained with aceto-orcein. In Fig. 19, the extremely extended vegetative nucleus is seen passing by the germ cell with the nucleus at mitotic metaphase. A scale indicates $20 \mu \mathrm{m}$ for Figs. 18 and 19 , and $10 \mu \mathrm{m}$ for other figures. 


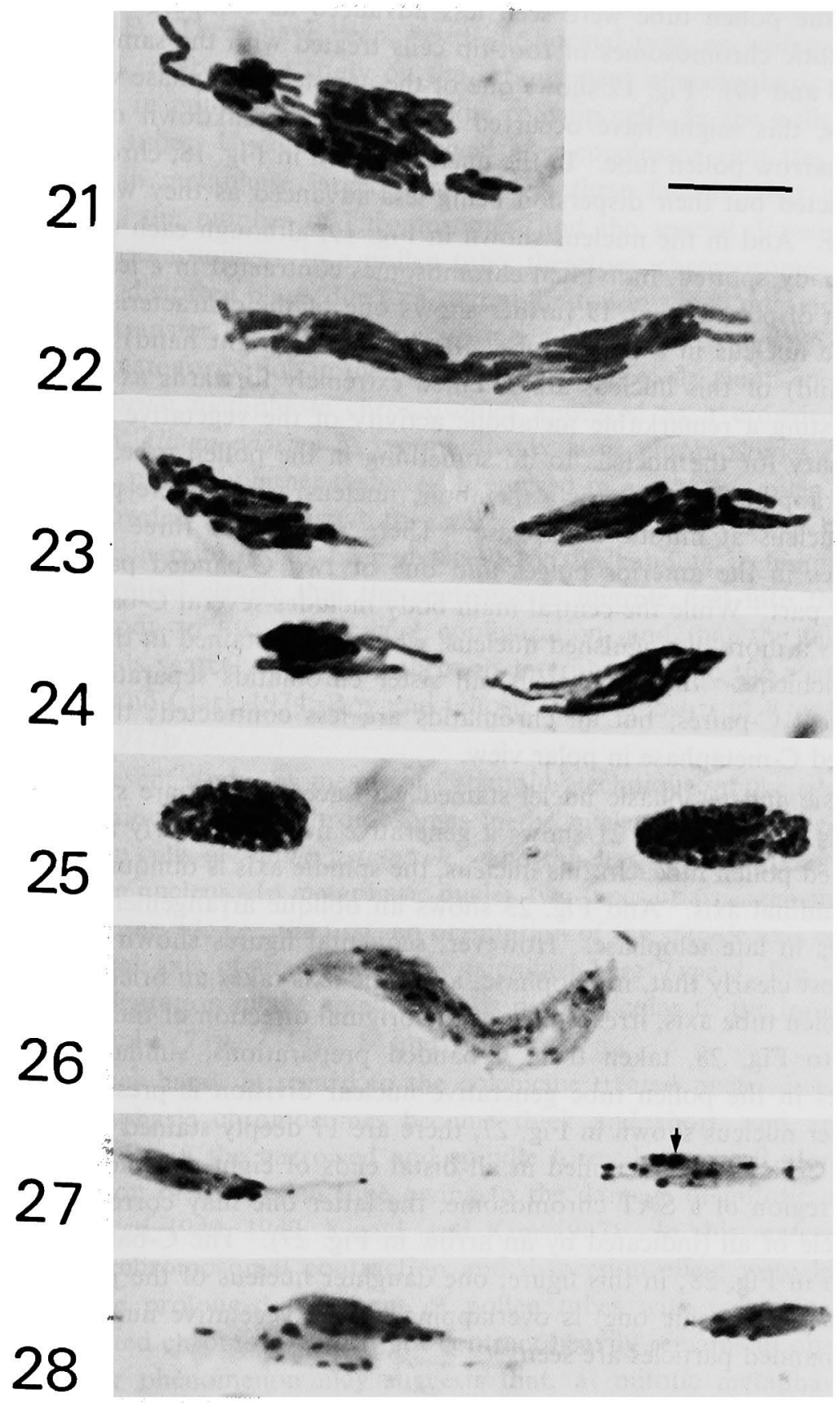

Figs. 21-28. Generative nuclear mitotic phases from early anaphase through late telophase. 21-25, stained with aceto-orcein. 26-28, stained by C-banding technique. 21,22 and 26 are anaphasic configurations; in which whole haploid chromosomes $(n=8)$ are separating along the pollen tube direction. 21, early anaphase. 22 and 26, mid-anaphase. In Fig. 27, C-banded particles are seen in the distal ends of every separating chromatids and in the satellite region of one of a SAT chromosome (indicated by an arrow). In Fig. 28, about $14 \mathrm{C}$-banded particles are seen in the less stained vegetative nucleus. Bar, $10 \mu \mathrm{m}$. 
direction in the pollen tube were seen less advanced as compared with that observed in mitotic chromosomes of root-tip cells treated with the same medium for $3 \mathrm{hr}$ (Figs. 18 and 19). Fig. 17 shows one of the arrested metaphase with dispersed chromosomes, this might have occurred by complete breakdown of the spindle body in the narrow pollen tube. In the nucleus shown in Fig. 18, chromosomes are rather contracted but their dispersion being less advanced as they were enveloped in the spindle. And in the nucleus shown in Fig. 19, although each sister chromatids have already splitted, individual chromosomes contracted in a less degree, and they were not dispersed. Fig. 19 further shows one of the characteristic feature of the vegetative nucleus in a pollen tube. Both anterior (right hand) and posterior parts (left hand) of this nucleus are extented extremely forwards as well as backwards, suggesting a remarkable metabolic activity of the vegetative nucleus when it was necessary for the nucleus to do something in the pollen tube. The vegetative nucleus, apparently acting as a metabolic nucleus, is seen here passing by the generative nucleus at mitotic metaphase. There are two or three heavy stained small particles in the anterior bulges and one or two C-banded particles in the posterior tail part. While the central main body includes several $\mathrm{C}$-banded regions. Fig. 20 shows a more distinguished nucleus, which was obtained in the $6 \mathrm{hr}$ culture including colchicine. In this nucleus, all sister chromatids separated completely forming typical $\mathrm{C}$-paires, but all chromatids are less contracted; this is a somewhat distorted C-metaphase in polar view.

Anaphasic and telophasic nuclei stained with aceto-orcein are shown in Figs. $21,22,23,24$ and 25 . Fig. 21 shows a generative nucleus at early anaphase seen in a broadened pollen tube. In this nucleus, the spindle axis is oblique to the pollen tube's longitudinal axis. And Fig. 23 shows an oblique arrangement of daughter chromosomes in late telophase. However, sequental figures shown in Fig. 23 to Fig. 25 suggest clearly that, in telophase, a spindle axis takes an orientation always along the pollen tube axis, irrespective of the original direction of the nuclear plate. In Fig. 26 to Fig. 28, taken from C-banded preparations, similar behavior of chromosomes in the pollen tube generative nuclear division is presented. In the right daughter nucleus shown in Fig. 27, there are 17 deeply stained particles; suggesting that $\mathrm{C}$-bands are identified in all distal ends of eight chromosomes and in the satellite region of a SAT chromosome, the latter one may corresponds to the largest particle of all (indicated by an arrow in Fig. 27). The C-banding patterns are also seen in Fig. 28; in this figure, one daughter nucleus of the just underwent nuclear division (left side one) is overlapping on the vegetative nucleus, in which about $14 \mathrm{C}$-banded particles are seen.

\section{Discussion}

In the 1930s and 1940s, one of the most debated problem concerning the generative cell division carried out inside the developing pollen tube has been that whether the metaphasic chromosomes form a regular equatorial plate or not (Maheshwari 1949). Cooper (1936) reported their equatorial alignment in Lilium (normal type), but various observations were reported in different plants species 
by many workers (Upcott 1936, Raghavan et al. 1939, Sax and O'Mara 1941, Johnston 1941). There have been, besides a normal type, an oblique and twisted type. After a comparative study on the arrangement of metaphasic chromosomes as observable in mitosis took place in the root-tip cells, in the pollen grains, and in the pollen tubes, Upcott (1936) arrived at a conclusion that the chromosomal arrangement in metaphase largely depends on three factors, that is, the size of chromosomes, the number of chromosomes, and the spacial dimension available for mitotic chromosomes. In a pollen tube, therefore, chromosomes tend to form an irregularly distorted plate, due to a spatial limitation which does not allow a free and smooth transfer of chromosomes to a nuclear plate. Ôta (1957) observed a normal type plate formation in the pollen tube mitosis of six plants species, but only in Allium odorum metaphasic chromosomes appeared as a crowded entity. $\mathrm{He}$ stated that in Allium odorum as comparatively large chromosomes rushed to the nuclear plate, probably either distorted or curved in a narrow pollen tube, so they formed a crowded metaphase. In recent years, however, the behavior of the chromosomes in mitosis has been detected by the banding technique and it has been demonstrated clearly that the centromeres migrate first into the equator of the spindle body to form metaphase configuration, and that the telophasic configuration holds scarcely unaltered through interphase up to the next mitotic prophase (Stack and Clark 1974, Roy and Ghosh 1977, Ghosh and Roy 1977, Tanaka and Tanaka 1977).

In the present study, by means of C-banding technique, above situation (topographical continuity of the chromosomes in the nucleus) has also been confirmed true in root-tip cells of Allium fistulosum. And this has been observed in the pollen tube generative nucleus. In metaphasic nuclei, two types of arrangement of chromosomes were observed; i.e., the first, an orientation of the spindle axis coincides with the longitudinal axis of the pollen tube (signified here Type 1, Fig. 16), and the second, an orientation of the spindle axis is perpendicular to the longitudinal axis of the pollen tube (Type 2, Figs. 14 and 15).

On the other hand, in regard to the colchicine treated nuclei, it is well known that the metaphasic chromosomes become thick and short, and arrange themselves dispersedly in the narrowed and spindle formed germ cell along the longitudinal direction of the pollen tube owing to the damage of mitotic apparatus by colchicine (Eigsti 1940, 1942, Kwack and Kim 1967). In this material, however, the degree of chromosomal contraction and dispersion effect were less advanced in spite of the prolonged treatment of pollen tubes with colchicine. Why the colchicine treated chromosomes do not contract heavily remains in obscure. However, the latter phenomenon may suggests that, at mitotic metaphase, the germ cell of this material is not so large enough to permit the chromosomes to arrange dispersedly. It is probably that the germ cell has a long spindle form in a narrow pollen tube at first, but by and by it becomes shorter and thicker to get an oval-like form as it prepares to enter nuclear division. And at metaphase, it becomes to get an oval-like form. This is a common feature for free cells in culture medium in vitro. The germ cell in question is just comparable to a free cell wandering in the pollen tube cytoplasm. And when the germ cell goes into mitosis, it 
takes an oval-like form which could have a wholeround direction in the pollen tube. But when a spindle body starts to extend, the mitotic axis may inevitably rotate in any direction in order to get the most stable situation in the pollen tube. In Type 1, the separation of chromosomes occurs in regular fashion with no apparent disturbance. In Type 2, however, it is inevitably needed for separation of chromosomes that two poles of the spindle body slide away in the opposite direction in the pollen tube (Figs. 21 and 23). Thus it seems reasonable that the metaphasic germ cell could take by chance either Type 1 or Type 2, and transitional situation available. This simple but important character may serve to realize the chromosome behavior in mitosis in the pollen tube of Allium fistulosum.

\section{Summary}

Behavior of the generative nuclear chromosomes in the cultured pollen tube in Allium fistulosum have been studied. The mitotic phases from mid-prophase to late-prophase and that from metaphase to telophase were observed in the 2 and the $4 \mathrm{hr}$ cultures, respectively. By means of $\mathrm{C}$-banding technique, it became clear that the constitutive nuclear topograph has been held invariable through telophase of the primary microspore division in a pollen grain to prophase of the secondary generative nuclear division in a pollen tube. Free spindle-formed germ cell in the pollen tube cytoplasm changes itself into an oval-like form before going into the secondary generative nuclear division. It seems very reasonable that thus formed mitotic germ cell could have a wholeround directional mitotic axis in the pollen tube. Usually mitotic axis seems to have a right angle orientation against the pollen tube membrane. But when the spindle body prepares to extend, the mitotic axis may inevitably rotates to get the most stable orientation in the pollen tube. The vegetative nucleus (=the pollen tube nucleus) usually wanders near-by the germ cell, but sometimes, it changes its form into a filamentous, extremely extended form as seen in Fig. 19. The reason why such transformation does occur remained to be studied.

\section{Acknowledgement}

The authors wish to express their gratitude to Professor Humihiko Ono for his continued interest and support.

This work was supported by Research Grant No. 334040 from the Ministry of Education of Japan.

\section{References}

Cooper, D. C. 1936. Development of the male gametes of Lilium. Bot. Gaz. 98: 169-177.

Eigsti, O. J. 1940. The effects of colchicine upon the division of the generative cell in Polygonatum, Tradescantia, and Lilium. Amer. J. Bot. 27: 512-524.

- 1942. A cytological investigation of Polygonatum using the colchicine-pollen tube technique. Amer. J. Bot. 29: 626-636.

Ghosh, S. and Roy, S. Ch. 1977. Orientation of interphase chromosomes as detected by Giemsa 
C-bands. Chromosoma (Berl.) 61 : 49-55.

Johnston, G. W. 1941. Cytological studies of male gamete formation in certain angiosperms. Amer. J. Bot. 28 : 306-319.

Kamizyô, A. and Tanaka, N. 1978. Studies on the generative nuclear division I. Comparative analysis of somatic chromosomes in Allium fistulosum by means of $\mathrm{C}$ - and Q-banding patterns. Cytologia 43: 679-688.

Kwack, B. H. and Kim, I. H. 1967. An improved method for culturing Tradescantia pollen tubes for chromosomal analysis. Cytologia 32: 1-5.

Maheshwari, P. 1949. The male gametophyte of angiosperms. Bot. Rev. 15: 1-75.

Ôta, T. 1957. Division of the generative cell in the pollen tube. Cytologia 22: 15-27.

Raghavan, T. S., Venkatasubbaan, K. R. and Wulff, H. D. 1939. Division of the generative cell in Impatiens balsamina L. Cytologia 9: 389-392.

Roy, S. Ch. and Ghosh, S. 1977. Orientation of Giemsa C-bands in interphase cells in Allium cepa L. Experientia 33: 432-433.

Sax, K. and O'Mara, J. G. 1941. Mechanism of mitosis in pollen tubes. Bot. Gaz. 102: 629-636.

Stack, S. M. and Clark, C. R. 1974. Chromosome polarization and nuclear rotation in Allium cepa roots. Cytologia 39: 553-560.

Tanaka, N. R. and Tanaka, N. 1977. Behavior of the differentially stained kinetochores during the mitotic cell cycle in some Polygonatum species (Liliaceae). Cytologia 42: 765-775.

Upcott, M. 1936. The mechanics of mitosis in the pollen-tube of Tulipa. Proc. Royal. Soc. London B. 121 : 207-220. 